promote appropriate goals such as furthering high quality medicine, patient centred care, and efficient use of resources. ${ }^{11}{ }^{11}$ The difficulty is to avoid or minimise the perverse side effects of incentives. That goal requires careful use of incentives, evaluation of their effect, and a fair dose of scepticism. Health policy makers in much of the world today embrace uncritically the use of incentives for doctors, ignoring their problems and risks. Instead they should treat incentives like drugs-a powerful product that can be beneficial but also dangerous. Society should control the use of incentives for doctors carefully to ensure that they are safe as well as effective in the way they are used.

Marc A Rodwin professor of law

Suffolk University Law School, 120 Tremont Street, Boston,

MA 02108 , USA

(mrodwin@suffolk.edu)

Competing interests: None declared.
1 Shaw GB. Preface on doctors. The doctor's dilemma: a tragedy. Baltimore: Penguin, 1913:9.

2 Lambert TW, Goldacre MJ, Vallance E, Mallick N. Characteristics of consultants who hold distinction awards in England and Wales: database analysis with particular reference to sex and ethnicity. BMJ 2004;328:1347-9.

3 Arrow KJ. Social responsibility and economic efficiency. Public Policy 1973;16:303-17.

4 Rodwin MA. Medicine, money and morals: physicians' conflicts of interest. New York: Oxford University Press, 1993.

5 Rodwin MA. Strains in the fiduciary metaphor: Divided physician loyalties and obligations in a changing health care system. Am J Law Med 1995;21:241-57.

6 Gold M, Hurley R, Lake T, Ensor T, Berenson R. A national survey of the arrangements managed care plans make with physicians. $N$ Engl J Med $1995 \cdot 3233 ; 1678-83$

7 Kassirer JP. On the take: how big business is corrupting American medicine. New York. Oxford University Press (in press).

8 Angell M. The truth about the drug companies-how they deceive us and what to do about it. New York: Random House (in press).

9 Krimsky S. Science in the private interest: has the lure of profits corrupted the virtues of biomedical research. Lanham: Roman and Littlefield, 2003.

10 Stevens S. Reform strategies for the English NHS. Health Affairs 2004;23:37-44.

11 Department of Health. General medical services (GMS) contract 2004 www.dhgov.uk/policyAndGuidance/HumanResourcesAndTraining/ ModernisingPay/GPContracts/fs/en (accessed 11 Mar 2004).

\title{
Scars and keloids
}

\section{Several treatments are used, but the evidence base is lacking}

$\mathrm{T}$ he reparative response of a fetus to injury is regeneration of tissue without scar. However, in children and adults the inevitable response to injury is scar formation, which in skin causes disfigurement and may result in restriction of motion. In other organs excessive scarring is responsible for pulmonary fibrosis, cirrhosis, end stage glomerulonephritis, and systemic scleroderma. The molecular signals that cause an active wound healing process to turn off in the process of scar maturation are unknown. The clinical treatment of scars has therefore been largely empirical. Multiple treatments have been proposed, often backed by anecdotal evidence only. Some treatments, such as topical vitamin $\mathrm{E}$, have been widely promulgated as effective in the popular press in the United States, whereas others have been marketed directly to the consumer despite a lack of evidence. ${ }^{2}$ So how should we treat excessive scarring given the poverty of evidence?

The first step in minimising scarring should be attention to the early care of wounds, and the following recommendations are based on general principles of wound healing. The goal with minor wounds such as abrasions is to achieve rapid epithelisation by moist healing with ointment or semiocclusive dressings. When epithelisation is delayed beyond 10-4 days the incidence of hypertrophic scarring goes up dramatically. ${ }^{3}$ Surgical closure of an open wound should take into account the tension on the wound. Wounds subjected to tension due to motion, body location, or loss of tissue (after excision of a lesion) are at increased risk of scar hypertrophy and spreading. Appropriate splinting of the tissue with permanent intradermal sutures should be considered. A useful technique is a subcuticular closure with a polyprolene suture that can be left in place for six months. Permanent clear nylon sutures placed in the deep dermis are also efficacious (personal experience). Most absorbable sutures, which do not splint the wound sufficiently long to prevent widening or hypertrophy of scar. ${ }^{4}$

Recently, an international group of clinicians reviewed the available literature for an evidence based analysis of useful treatments for cutaneous scarring and, where evidence was lacking, a consensus recommendation of useful approaches. ${ }^{3}$ For the prevention of hypertrophic scarring and optimal scar formation after laceration or surgical incision, a semiocclusive tape or ointment, applied for one to four weeks, has been accepted as useful, although the only evidence is from successful clinical experience. ${ }^{3}$ The underlying mechanism has not been well elucidated, and the evidence is circumstantial. Epithelisation over an open wound induces apoptosis and resolution of inflammation, and in co-culture in vitro experiments signals from overlying keratinocytes resulted in reduced synthesis of collagen by the underlying fibroblasts. ${ }^{5}$ In addition, hydration of the keratinocytes (similar to hydration of epithelium by a semiocclusive tape) results in cytokine signalling from the keratinocytes, which influences fibroblasts in co-culture experiments. ${ }^{6}$

In patients with scars showing early signs (erythema and scar elevation) of evolving into hypertrophic scars, or for patients at higher risk of developing hypertrophic scars (patients who are younger than 40 years, have a previous history of hypertrophic scars, or are at increased risk because their racial background), several prospective randomised studies support the efficacy of silicone gel sheeting in flattening scars and reducing their stiffness. ${ }^{3}$ Although the mechanism of action has not been proved, multiple studies have by elimination assumed that its action is the result of its semiocclusive properties. ${ }^{367}$ In recent years multiple silicone gel

Competing interests on bmj.com 
products have been marketed with the implication that they are equivalent to silicone gel sheeting in efficacy. ${ }^{8}$ However, no comparative clinical trials have been performed on these products.

For scars that fail to respond to silicone gel sheeting several prospective studies show the efficacy of local injections of insoluble steroid (triamcinolone). ${ }^{39}$ No good evidence exists to support the efficacy of topical steroids, presumably because of a lack of penetration into the underlying deep dermis.

Surgical excision of hypertrophic scars may be efficacious but requires meticulous adherence to the surgical principles and adjunctive measures discussed above.

Laser treatment for scars has received considerable media attention. Multiple different laser wavelengths and treatment protocols have been proposed, and anecdotal evidence supports their use. ${ }^{3}$ However, without prospective randomised studies we cannot know whether laser treatment accelerates the normal process of scar resolution or actually results in long term improvement.

Burn scars, and hypertrophic scars that develop from other open wounds with delayed epithelisation, also respond to silicone gel sheeting and steroids, but local pressure achieved with elastic garments has achieved widespread acceptance since its was first described 30 years ago, despite a lack of favourable prospective randomised trials. ${ }^{9}$

Several therapeutic options for the treatment of scars may become available in the next few years. Several pharmaceutical companies are engaged in research, to identify novel targets for therapy. Experimental evidence implicates the importance of members of the transforming growth factor $\beta$ family in cutaneous scarring, as well as scarring in other organs. ${ }^{10}$ A number of different approaches have been taken to modify activity of transforming growth factor $\beta$ locally. ${ }^{11}$ The other principal strategy being undertaken by pharmaceutical companies is to interfere with collagen synthesis locally, and this has been effective in an animal model. ${ }^{12}$
Keloids represent the most extreme example of cutaneous scarring and are the most difficult to treat. Many papers blur the definition of a keloid (which grows in a progressive fashion beyond the borders of the initial injury) and hypertrophic scars (which are self limiting in their growth). Many keloids are unresponsive to silicone gel sheeting or steroids, and radiation therapy (1200-2000 gy in five doses) has been used with success, although the risks have made some clinicians avoid it altogether. ${ }^{3}$ An intriguing approach to treat difficult scars and keloids in small uncontrolled series is the use of local chemotherapeutic agents, such as bleomycin and 5-fluorouracil.

\section{Thomas A Mustoe professor}

Division of Plastic Surgery, Feinberg School of Medicine, Northwestern University, Chicago, Chicago Ill 60611, USA (tmustoe@nmh.org)

1 Havlik RJ. Vitamin E and wound healing: safety and efficacy reports. Plast Reconstruct Surg 1997;1901-2.

2 Saulis AS, Mogford JA, Mustoe TA. The effect of Mederma ${ }^{R}$ on hypertrophic scarring in the rabbit ear hypertrophic scar model. Plast Reconstr Surg 2002;110:177-83.

3 Mustoe TA, Cooter R, Gold M, Hobbs R, Ramelet AA, Shakespeare P, et al. International clinical guidelines for scar management. Plast Reconstr Surg 2002:110:560-72.

4 Elliot, D, Mahaffey PJ. The stretched scar: the benefit of prolonged dermal support. Br Journal Plastic Surg. 42:74-78, 1989.

5 Brown DL, Kao WW, Greenhalgh DG. Apoptosis down-regulates inflammation under the advancing epithelial wound edge: delayed patterns in diabetes and improvement with topical growth factors. Surgery 1997;121:372-80.

6 Chang CC, Kuo YF, Chiu HC, Lee JL, Wong TW, Jee SH. Hydration, not silicone, modulates the effects of keratinocytes on fibroblasts. J Surg Res 1995;59:705-11.

7 Saulis AS, Chao J, Telser A, Mogford JE, Mustoe TA. Silicone occlusive products in the treatment of hypertrophic scar in the rabbit ear hypertrophic scar model. Aesthetic Surg J 2002;22:147-53.

8 Ahn ST, Monafo, W, Mustoe TA. Topical silicone gel: a new treatment for hypertrophic scars. Surgery 1989;106:781-7.

9 Chang P.Laubenthal KN, Lewis RW 2nd, Rosenquist MD, Lindley-Smith P, Kealey GP. Prospective, randomized study of the efficacy of pressure garment therapy in patients with burns. J Burn Care Rehabil 1995; $16: 473-5$.

10 Blobe GC, Schiemann WP, Lodish HF. Role of transforming growth factor-Beta in human disease. N Engl J Med 2000; 342:1350-8.

11 Shah M, Foreman DM, and Ferguson MW. Neutralization of TGF- $\beta 1$ and TGF- $\beta 2$ or exogenous addition of TGF- $\beta 3$ to cutaneous rat wounds reduces scarring.J Cell Sci 1995;108:985-1002.

12 Kim I, Xia Y, Mogford J, Witschi C, Nafissi M, Mustoe TA. Collagen prolyl 4 hydroxylase inhibitor reduces scar elevation in a rabbit ear model of hypertrophic scarring. Surg Forum 2000;51:589-91.

\section{Sentinel lymph node biopsy}

Is now an established and widely available technique for breast cancer and melanoma

$\mathrm{S}$ entinel lymph node biopsy has developed over the past decade as a minimally invasive technique to assess regional lymph node status in patients with malignancy. This technique allows us to find out the status of a lymph node basin by removing only a small number of nodes. These nodes stand sentry to the rest of the nodal basin: if malignant disease is going to affect a nodal region it must first pass through the sentinel node. Therefore the nodal basin will contain malignant cells only if the sentinel node is first involved. The specific and limited removal of the sentinel node reduces surgical insult and morbidity compared with conventional lymphatic clearance. Sentinel lymph node biopsy is now widely available, and most cancer surgeons offer this as part of their diagnostic protocol for patients.
Sentinel lymph node biopsy was initially developed to detect lymphatic metastasis in parotid carcinoma. ${ }^{1}$ As this technique has developed it has been used in the management of penile carcinoma, but it is now predominantly used in the diagnosis of lymphatic metastasis from breast cancer and melanoma. ${ }^{2-4}$ It can also be used to detect extent of spread of a wide range of gastrointestinal and endocrine tumours. ${ }^{5}$ The shortcoming of this technique in these diseases is that primary surgery with sentinel lymph node biopsy, though feasible, may not be practical: a second surgery for lymphatic clearance for pancreatic or gastrointestinal malignancy is less practical and a greater surgical

Additional references w1-w3 are on bmj.vom 